September 4, 2005

\title{
The peculiar optic, dielectric and x-ray diffraction properties of a fluorinated de Vries asymmetric-diffuse-cone-model ferroelectric liquid crystal
}

\author{
Jan P. F. Lagerwall ${ }^{1 *}$, David Coleman², Eva Körblova ${ }^{3}$, Chris Jones ${ }^{2}$, Renfan \\ Shao ${ }^{2}$, José M. Otón ${ }^{4}$, David M. Walba ${ }^{3}$, Noel Clark ${ }^{2}$, and Frank Giesselmann ${ }^{1}$ \\ ${ }^{1}$ Institute of Physical Chemistry, University of Stuttgart, \\ Pfaffenwaldring 55, D-70569 Stuttgart, Germany \\ ${ }^{2}$ Department of Physics, Condensed Matter Laboratory, \\ University of colourado, Boulder, CO 80309, USA \\ ${ }^{3}$ Department of Chemistry and Biochemistry, \\ University of colourado, Boulder, CO 80309, USA \\ ${ }^{4}$ Dept Tecnología Fotónica, ETSI Telecomunicacin, \\ Univ. Politécnica de Madrid, E-28040 Madrid, Spain
}

* E-mail for correspondence: jan.lagerwall@ipc.uni-stuttgart.de Fax: 00497116842569

Telephone: 00497116854458 


\begin{abstract}
A newly synthesised semi-fluorinated chiral smectic liquid crystal, W504, is investigated by electrooptic, dielectric and x-ray scattering experiments. It exhibits a huge dielectric soft mode response, strong electroclinic effect and a birefringence which increases considerably with the director tilt angle $\theta$, typical characteristics of a $\mathrm{SmA}^{*}{ }_{-} \mathrm{SmC}^{*}$ transition following the de Vries asymmetric diffuse cone $(\mathrm{ADC})$ model in which the non-zero director tilt in $\mathrm{SmC}^{*}$ arises through an ordering of tilting directions rather than an actual increase in average molecule tilt $\left\langle\theta_{\text {mol }}\right\rangle$. In W504 a small increase in $\left\langle\theta_{\text {mol }}\right\rangle$ of about $4^{\circ}$ is however detected in the $\mathrm{SmC}^{*}$ phase. Although the increase in molecule inclination is much less than the increase in director tilt $\theta$, saturating close to $30^{\circ}$, it leads to a shrinkage of the smectic layers by about $1 \AA$, a result of the large initial molecule tilt in SmA* , $\left\langle\theta_{m o l}\right\rangle_{S m A *} \approx 30^{\circ}$. The tilting transition in W504 is thus mainly an ADC model disorder-order transition, but it also has a component of a structural transition. The semi-fluorinated molecular structure of W504 leads to a very weak electron density modulation along the layer normal, giving a vanishing form factor in bulk samples which exhibit no (001) x-ray scattering peak. In thin films the (001) peak is however observed, indicating that the electron density modulation is enhanced by the breaking of the head-tail symmetry of the liquid crystal phase at the LC-air interface.
\end{abstract}




\section{INTRODUCTION}

In the research on ferroelectric and antiferroelectric liquid crystals (FLCs and AFLCs), i.e. materials exhibiting the chiral smectic-C $\left(\mathrm{SmC}^{*}\right)$ phase or one or more of its variations, considerable attention has over the last few years been directed towards compounds exhibiting the unusual property of a smectic layer thickness which is essentially independent of the director tilt angle $\theta[1-14]$. In the $\mathrm{SmC}^{*}$ phase the director $\mathbf{n}$ is inclined by an angle $\theta>0$ with respect to the smectic layer normal, $\mathbf{k}$, a geometry which in combination with chirality results in a spontaneous polarization $\mathbf{P}_{s}$ along $\mathbf{n} \times \mathbf{k}[15]$. Normally, the layers contract below the transition from $\mathrm{SmA}^{*}$ - where $\theta \equiv 0$ in absence of fields - to $\mathrm{SmC}^{*}$ or one of its AFLC variations. In some compounds this shrinkage is much smaller than expected, a behaviour which was explained by the Dutch-American crystallographer Adriaan de Vries. He recognised that one must take into account that the molecules have a non-zero average inclination $\left\langle\theta_{\text {mol }}\right\rangle$ away from $\mathbf{k}$ even if $\theta=0$, as in $\mathrm{SmA}^{*}$, a result of the orientational disorder characteristic of any LC phase [16-19]. The uniaxial symmetry around $\mathbf{k}$ and the absence of director tilt are consequences of the fact that all directions of molecule inclination are equally probable in the orientational fluctuations.

In de Vries' scenario for the SmA-SmC transition without layer shrinkage $\left\langle\theta_{\text {mol }}\right\rangle$ remains constant, while the increasing director tilt $\theta>0$ comes about through a gradual azimuthal biasing towards a certain molecule inclination direction. He referred to this model as the 'asymmetric diffuse cone' (ADC) model [19], the asymmetry being equivalent to the azimuthal biasing present in the SmC phase. The 'diffuse cone' can be seen as the trajectory over time of a single molecule fluctuating around $\mathbf{n}$ in any LC phase. Recent analysis of the ADC model has shown that, in the case of chiral smectics, it implies that the birefringence $\Delta n$ strongly increases with $\theta[5]$ and that the $\mathrm{SmA}^{*}$ electroclinic effect and soft mode (fieldinduced tilt $\theta>0$ and the corresponding polarization fluctuation) are unusually large [1, 20]. These properties have indeed been identified in a number of compounds with minimal layer shrinkage.

So far, ADC behaviour has been found mainly in mesogens that have at least one terminal chain quite different from standard alkyl chains, e.g. heavily fluorinated chains $[2,6-8,10,11,13]$, with a siloxane moiety attached $[4,5,9]$, or even with the mesogens attached to a polysiloxane backbone $[12,14]$. In this paper we describe x-ray scattering, 
dielectric and electrooptic investigations of a new FLC (Figure 1) of the former class, exhibiting several of the properties typical of ADC materials to a very strong degree, for instance a huge dielectric response. However, its x-ray scattering response is quite peculiar. Not only does the compound exhibit stronger layer shrinkage than most ADC materials investigated so far, but the first-order x-ray scattering peak is essentially undetectable when studying bulk samples. The new results make it clear that the $\mathrm{SmA}^{*}$-SmC ${ }^{*}$ transition can involve several mechanisms in parallel, and that even when the ADC model mechanism is strongly dominant it is not sufficient for achieving temperature-independent smectic layer thickness.

\section{INSERT FIGURE 1 ABOUT HERE}

\section{EXPERIMENTAL}

The liquid crystal compound, W504 (synthesised in the Boulder chemistry lab), was filled into cells with three different types of alignment layers: standard commercial polyimidecoated EHC cells, hard-buffed polyimide-coated cells from the Chalmers MC2 cell production line (Gothenburg, Sweden) and nylon-coated cells made in the Madrid cleanroom. The cells were kept in Instec mK2 or Julabo FP-25 hot stages for temperature control. Sample texture observations and optic and electrooptic measurements were done using an Olympus BH-2 polarizing microscope. The optical tilt angle $\theta$ and birefringence $\Delta n$ were measured with high resolution using a temperature scanning technique developed by Saipa and Giesselmann [21]. The magnitude of the spontaneous polarization $P_{s}$ was measured by integrating the polarization reversal current while switching the sample with a triangular waveform electric field [22]. Dielectric spectroscopy with simultaneous sample texture monitoring was performed using an HP4192A impedance bridge, a USB video camera (Logitech) and DiScO measurement software (FLC Electronics).

Three series of X-ray diffraction (XRD) experiments were carried out, in two locations. In the Stuttgart lab $\left(\mathrm{Cu}-K_{\alpha}\right.$ generator) the small-angle scattering (SAXS) from unaligned samples in $0.7 \mathrm{~mm}$ diameter Mark capillary tubes was investigated with a Kratky compact camera and a 1D position sensitive detector (A. Paar), and the wide-angle scattering 
(WAXS) pattern from samples aligned by a $2.5 \mathrm{~T}$ magnetic field was determined using a flat film camera loaded with digital imaging plates (Fuji BAS-SR system). With the National Synchrotron Light Source at Brookhaven National Laboratory (beamline X10A) experiments were carried out in reflectivity mode on samples aligned homeotropically on an octadecyltriethoxysilane self-assembled monolayer (OTE SAM) as well as in transmission mode on capillary samples.

\section{RESULTS AND DISCUSSION}

\section{A. Textures and electrooptic behaviour}

Similar to other fluorinated ADC compounds [8] W504 is difficult to align in standard LC sample cells. Figure 2 shows representative textures in the $\mathrm{SmA}^{*}$ and $\mathrm{SmC}^{*}$ phases in the three different cell types used. Nylon has previously been shown to be one of the best alignment materials for this type of compounds [23] and, indeed, the nylon-coated cell produced excellent uniform alignment in the $\mathrm{SmA}^{*}$ phase (Figure 2a). As the sample was cooled into $\mathrm{SmC}^{*}$, however, both domain types (corresponding to the two possible in-plane director tilt directions) developed a director structure with a strong twist from substrate to substrate. This led to poor maximum extinction and to washed-out colours, changing with sample orientation and not reflecting the birefringence, in bright orientations, cf. Figure $2 \mathrm{~b}$ and c. This cell was thus not suitable for measurements of the optical properties of W504.

\section{INSERT FIGURE 2 ABOUT HERE}

The $\mathrm{SmC}^{*}$ twist was much less pronounced in cells coated with polyimide (PI) but the standard commercial cells (EHC, row 2 in Figure 2) did not impose a uniform direction of the smectic layer normal, rendering quantitative measurements impossible also with this cell. However, it is qualitatively clear already from the colour change from yellow to orange on cooling the $4 \mu \mathrm{m}$ sample from $\mathrm{SmA}^{*}$ (d) to non-helical $\mathrm{SmC}^{*}$ (e and f) that $\Delta n$ increases substantially in $\mathrm{SmC}^{*}$, as expected for $\mathrm{ADC}$ materials. In the (partially) helical regions the colour remained yellow, a result of the helical averaging of the optic axis direction decreasing the effective value of $\Delta n$. The yellow-orange colours also reveal that $\Delta n$ is lower than in 
most smectic compounds, which in a $4 \mu \mathrm{m}$ cell typically exhibit colours between red and blue, i.e. at the border between first and second order in the Michel-Levy interference colour chart.

By using strongly buffed PI cells, we could achieve a sufficiently homogeneous alignment and at the same time avoid the director twist in $\mathrm{SmC}^{*}$, as shown in the two lowest rows in Figure 2. Decreasing the cell gap to $1.5 \mu \mathrm{m}$ a relatively defect-free alignment was obtained (j - l), allowing quantitative measurements of $\Delta n$ and $\theta$. The thin cell gap also assured complete helix and twist expulsion even in the absence of fields, allowing for measurements also in the field-free state. The results are shown in Figure $3 \mathrm{a}$ and b. In addition to the remarkably low values of birefringence, the typical ADC characteristic of $\Delta n$ increasing with $\theta$ stand out from these measurement results. The birefringence at the low-temperature limit of $\mathrm{SmC}^{*}$ (the first measurements were taken in supercooled $\mathrm{SmC}^{*}$ ) is about $22 \%$ larger than in field-free $\mathrm{SmA}^{*}$. As is also typical of ADC materials, the magnitude of the electroclinic effect is very large, with an induced tilt of $\theta \approx 10^{\circ}$ about $5^{\circ} \mathrm{C}$ above the $\mathrm{SmA}^{*}-\mathrm{SmC}^{*}$ transition, and a corresponding field-induced increase of $\Delta n$. Both the $\theta$ and the $\Delta n$ curves are continuous, without any discrete jump around the $\mathrm{SmA}^{*}{ }_{-} \mathrm{SmC}^{*}$ phase transition, which thus seems to be of second order. Indeed, DSC measurements at $10 \mathrm{~K} / \mathrm{min}$ heating and cooling rates could not resolve any transition peak, confirming the continuous nature of the transition.

\section{INSERT FIGURE 3 ABOUT HERE}

\section{B. Spontaneous polarization and dielectric response}

The value $P_{s}$ of the spontaneous polarization is shown as a function of temperature in Figure 3c. It has a negative sign and reaches the quite substantial value of $-200 \mathrm{nCcm}^{-2}$ (in supercooled $\mathrm{SmC}^{*}$ ). Considering the high magnitude of $P_{s}$ a quite strong dielectric response should be expected, but the actual dielectric spectra that we obtained on a $23.5 \mu \mathrm{m}$ planar-aligned (PI-coating) sample by far surpassed all expectations. At minimum measuring field strength, the dielectric loss $\epsilon^{\prime \prime}$ reached values above 500 in the $\mathrm{SmC}^{*}$ phase (Figure 4a), where the response can be attributed to a phason mode corresponding to helix 
distortion. This was however only about half of the maximum response, which was detected at the $\mathrm{SmA}^{*}-\mathrm{SmC}^{*}$ transition and can thus be attributed to the soft mode. Such behaviour is unheard of in normal FLCs, where the $\mathrm{SmC}^{*}$ phason mode is usually much stronger than the soft mode, but it was recently shown to be a characteristic of chiral ADC compounds [20]. The strong soft mode is a result of the very weak restoring force counteracting director tilt (and, consequently, polarization) fluctuations in the ADC mechanism. This peculiarity follows from the fact that the director tilt in $\mathrm{SmC}^{*}$ arises mainly through an azimuthal tilt direction ordering. With $\epsilon^{\prime \prime}$ almost reaching 1000 at the $\mathrm{SmA}^{*}{ }_{-} \mathrm{SmC}^{*}$ transition, the soft mode response of W504 is by far the strongest known to us, even being more than twice as strong as that of the ADC compound from 3M studied in reference [20]. This difference scales quite well with the difference in $\theta-P_{s}$ coupling constant, being two to three times smaller in the $3 \mathrm{M}$ compound than in W504.

\section{INSERT FIGURE 4 ABOUT HERE}

On increasing the field strength to $20 \mathrm{mV} / \mu \mathrm{m}$ - a quite typical field strength for lowamplitude dielectric spectroscopy investigations on FLCs - the phason mode absorption grew drastically in strength, raising $\epsilon^{\prime \prime}$ in $\mathrm{SmC}^{*}$ to values around 1600, thereby overshadowing the soft mode response at the phase transition, cf. Figure 4b. This very strong SmC* response arises because the measuring field induces not only helix distortion, but even helix unwinding commences. This was verified by studying the texture photos taken during the measurement, two examples of which are shown in the insets of Figure $4 \mathrm{a}$ and b. The periodic helix lines were easy to see in the photos taken during the $2 \mathrm{mV} / \mu \mathrm{m}$ scans, but partially or completely absent during the scans with $20 \mathrm{mV} / \mu \mathrm{m}$ measuring field strength. Such a low helix unwinding / switching threshold is very unusual among FLCs but it has been reported also for other ADC compounds, even in the case of AFLCs [7].

In order to make it clear just how huge the dielectric response of W504 is, an absorption spectrum of $(S)$-S1B8, a typical non-ADC FLC with similar $P_{s}$ (saturating at $-160 \mathrm{nCcm}^{-2}$; see Schacht et al. [24] for more details on this compound), is shown in Figure 4 c. The measurement was done in the same type of cell, with the same thickness, at 2 and $20 \mathrm{mV} / \mu \mathrm{m}$, giving essentially identical spectra. The data are plotted with the same scaling as in a and b. The phason and soft mode absorptions of S1B8, producing $\epsilon^{\prime \prime}$ values of about 50, are then 
hardly visible. (Actually, the main absorption seen in c, at low frequencies $(10-100 \mathrm{~Hz})$, is not due to the polar nature of the $\mathrm{SmC}^{*}$ phase but to a relatively large conductivity of the sample.)

\section{X-ray scattering results}

We studied the XRD profiles of W504 both in the small-angle and in the wide-angle regime. Examples of $\mathrm{SmA}^{*}$ and $\mathrm{SmC}^{*}$ radial SAXS profiles are shown in Figure 5a and $\mathrm{b}$ as a function of scattering vector magnitude $q$. During the measurements with $\mathrm{Cu}-K_{\alpha}$ radiation (a) we noted that the scattering intensity was generally very weak (exposure times 2-5 times longer than usual were used). The biggest surprise was however that no (001) pseudo-Bragg peak corresponding to the smectic layering could be resolved at any temperature above crystallization in the expected range of $q \sim 0.18$ (equivalent to a layer thickness of $\sim 35 \AA$ ). In the $\mathrm{SmA}^{*}$ phase a diffuse increase in intensity could be seen at about twice this scattering angle (which would correspond to the (002) peak) but this 'bump' was too small to do any reliable analysis. The very unusual absence of first-order smectic XRD peak suggests that the form factor is close to zero, a characteristic which most likely is related to the electron-rich fluorinated end chain of W504. Although only one chain is fluorinated, the head-tail symmetry in a bulk LC sample results in an equal number of molecules where this chain points mainly upwards and downwards, respectively, hence the effective electron density can be quite similar in core and tail regions. The unusually weak modulation in electron density along the layer normal could thus explain the vanishing form factor.

\section{INSERT FIGURE 5 ABOUT HERE}

With the well-aligned OTE-SAM sample investigated at the synchrotron source, diffraction profiles where the smectic layer peaks are easy to resolve could however be obtained, even though the intensity of the (001) peak was still much lower than that of (002), cf. Figure 5b. We speculate that the scattering in the case of the OTE-SAM sample is enhanced by the breaking of the head-tail symmetry at the LC-SAM and LC-air interfaces. Close to the sample surface we may expect a large difference in number of molecules aligned fluorine tail up and down, respectively. As it is the near-surface volume of the sample that contributes 
to the reflectivity measurement, this experiment would then sense a larger electron density contrast than during the experiment on bulk samples. Hence the form factor is non-zero, rendering the (001) peak visible.

In contrast, when repeating the bulk LC measurements on capillaries at the synchrotron, no (001) peak was visible but a weak (002) peak was observed, just as with the Kratky camera measurements. In Figure $5 \mathrm{c}$ we present the smectic layer spacing $d$ vs. temperature obtained from the capillary and OTE samples. While there appears to be a slight shift in the transition temperatures and in the $d$-spacing, the data are qualitatively equivalent indicating that the scattering peak observed in capillary samples is indeed the (002) peak of the smectic layering and that the form factor is approximately zero at the (001) peak. The issue of the varying scattering behaviour of W504 in different sample geometries is currently being investigated more carefully and the results will be reported in a future paper.

We note that the smectic layer spacing in $\mathrm{SmA}^{*}, \sim 35 \AA$, corresponds to an average tilt of the molecular long axis with respect to the layer normal, $\left\langle\theta_{\text {mol }}\right\rangle$, of about $26^{\circ}$ (using the molecule length obtained with MOPAC / AM1, cf. Figure1). Such a large average molecule tilt in the $\mathrm{SmA}^{*}$ phase is typical for ADC compounds [8, 9], whereas it in ordinary materials is $15-20^{\circ}$ [18]. On the other hand, the smectic layers in W504 exhibit a nonnegligible contraction below the $\mathrm{SmA}^{*}{ }_{-} \mathrm{SmC}^{*}$ transition which is somewhat of a surprise considering the very strong ADC characteristics observed in the electrooptic and dielectric response. To understand this we need to investigate the temperature variation of $\left\langle\theta_{\text {mol }}\right\rangle$. The best way to assess $\left\langle\theta_{m o l}\right\rangle$ is to analyse the directional (azimuthal) distribution of the wide-angle x-ray scattering-originating from the liquid-like intralayer molecular order-in the $\mathrm{SmA}^{*}$ and $\mathrm{SmC}^{*}$ phases.

The non-polar and uniaxial orientational order in e.g., a nematic or smectic-A liquid crystal is described by the orientational distribution function (ODF) $f(\beta)$ where $\beta$ denotes the angle between the long molecular axis and the director of the uniaxially ordered phase. Since $\mathbf{n}$ is co-linear with $\mathbf{k}$ in $\mathrm{SmA} / \mathrm{SmA}^{*}, \beta \equiv \theta_{\text {mol }}$ in this phase. The ODF is usually expanded in terms of Legendre polynomials with the expansion coefficients $\left\langle P_{2}\right\rangle,\left\langle P_{4}\right\rangle, \ldots$ defining the orientational order parameters $S_{2}, S_{4}$, etc. Experimentally, we can determine $f(\beta)$ and the related order parameters in the $\mathrm{SmA}^{*}$ phase by analyzing the azimuthal intensity profile $I(\chi)$ in the WAXS pattern. The angle $\chi$ indicates the scattering direction and we define it such that the magnetic field is directed along $\chi= \pm 90^{\circ}$. Since $\mathbf{n}$ in $\mathrm{SmA}^{*}$ 
aligns with the field, this is also the direction of $\mathbf{k}$.

For our analysis, we use a procedure developed by Davidson, Petermann, and Levelut [25] where the ODF is expanded in terms of $\cos ^{2 n} \beta$-functions:

$$
f(\beta)=\sum_{n=0}^{\infty} f_{2 n} \cos ^{2 n} \beta
$$

The corresponding WAXS intensity profile can also be written as a series of $\cos ^{2 n} \chi$ functions involving the same $f_{2 n}$,

$$
I(\chi)=\sum_{n=0}^{\infty} f_{2 n} \frac{2^{n} n !}{(2 n+1) ! !} \cos ^{2 n} \chi
$$

Hence, by fitting Eq. (2) to the experimental scattering profile $I(\chi)$, with the $f_{2 n}$ being the parameters to fit, $f(\beta)$ can directly be calculated by inserting the fitted $f_{2 n}$ into the expansion in Eq. (1). With $f(\beta)$ known, any average $\langle X\rangle$ of a certain property $X$ that is related to the orientational distribution can be numerically calculated, e.g. $S_{2}$, and $\left\langle\theta_{\text {mol }}\right\rangle$. In the $\mathrm{SmC}^{*}$ phase, however, the WAXS pattern includes the orientational averaging due to the helical superstructure, hence $S_{2}$ cannot be extracted (being uniaxially symmetric, the Legendre polynomial order parameters are not defined in the biaxial $\mathrm{SmC}^{*}$ phase).

In Figure 6 the WAXS intensities measured in $\mathrm{SmA}^{*}$ and $\mathrm{SmC}^{*}$ are plotted as a function of $\chi$, together with the corresponding best fits of Eq. 2. The resulting values of $\left\langle\theta_{\text {mol }}\right\rangle$ and $S_{2}$ are also given. We note that the orientational order determined in the $\mathrm{SmA}^{*}$ phase is remarkably low, $S_{2}=0.52$, corresponding to $\left\langle\theta_{m o l}\right\rangle_{S m A *} \approx 33^{\circ}$. This is a characteristic that W504 shares with previously investigated ADC materials [8, 9]. However, the value obtained with this method estimates $S_{2}$ downwards, i.e. the real (local) orientational order may be sligthly higher, and $\left\langle\theta_{\text {mol }}\right\rangle$ slightly smaller. This is because our measurement averages the order over a macroscopic volume, hence phenomena such as layer undulations and sample mosaicity contribute negatively to the measured value of $S_{2}$. However, the low $\Delta n$ values and the fact that the smectic layer spacing in $\mathrm{SmA}^{*}$ is much smaller than the expected molecule length suggest that the local orientational order indeed is low in W504.

\section{INSERT FIGURE 6 ABOUT HERE}

When cooling the sample to the $\mathrm{SmC}^{*}$ phase, the WAXS profile broadens somewhat, reflecting a small increase of average molecule inclination, at $110^{\circ} \mathrm{C}$ being close to $37^{\circ}$. This constitutes an important difference to the ADC material investigated in [8], where 
$d$ was largely unaffected by the $\mathrm{SmA}^{*}{ }_{-} \mathrm{SmC}^{*}$ transition and where the WAXS patterns in $\mathrm{SmA}^{*}$ and $\mathrm{SmC}^{*}$ could hardly be distinguished. Although the broadening in W504 is small, corresponding to an increase of $\left\langle\theta_{\text {mol }}\right\rangle$ of only about $4^{\circ}$, the large starting value of the molecular tilt angle gives the increase a considerable impact on $d$, about $5 \%$ shrinkage. Had $\left\langle\theta_{m o l}\right\rangle_{S m A *}$ been zero, the tilt increase would have had to be 18 degrees to produce such shrinkage. Taking $d$ in $\mathrm{SmA}^{*}$ to be about $35 \AA$ the WAXS analysis would thus predict a shrinkage of $1.8 \AA$, which is more than the actual shrinkage. This is another indication that the influence of layer undulations and sample mosaicity on the WAXS pattern produce apparent $\left\langle\theta_{\text {mol }}\right\rangle$ values which are too large. The relative increase of about $4^{\circ}$ should however be close to the real $\left\langle\theta_{m o l}\right\rangle$ increase. The much larger increase in the optical tilt angle $\theta$ displayed in Figure 3 is thus due to the strong ADC aspect of the $\mathrm{SmA}^{*}$-SmC* transition in W504, i.e. it can be attributed mainly to an ordering of tilting directions, in accordance with the electrooptic and dielectric behaviour of the compound.

\section{CONCLUSIONS}

The non-zero director tilt in the $\mathrm{SmC}^{*}$ phase of the semi-fluorinated mesogen W504 can be largely attributed to an ordering of tilting directions of molecules which exhibit a considerable tilt already in the $\mathrm{SmA}^{*}$ phase. This compound is thus quite well described by the asymmetric diffuse cone (ADC) model of Adriaan de Vries, as verified by a birefringence $\Delta n$ which increases strongly with director tilt $\theta$, strong electroclinic effect and huge dielectric soft mode response in the $\mathrm{SmA}^{*}$ phase, and a wide-angle x-ray diffraction pattern which changes relatively little between $\mathrm{SmA}^{*}$ and $\mathrm{SmC}^{*}$. However, in contrast to a number of other ADC smectics which have recently been investigated, there is a non-negligible layer shrinkage of about $1 \AA$ below the SmA*-SmC* transition in W504. This is a result of the large starting magnitude of the average molecule tilt with respect to the smectic layer normal in the SmA* phase, $\left\langle\theta_{m o l}\right\rangle_{S m A *} \approx 30^{\circ}$, giving even the small increase of about $4^{\circ}$ detected on cooling W504 into $\mathrm{SmC}^{*}$ a considerable impact on the layer spacing. Counter-intuitively, through the large average molecule tilt in $\mathrm{SmA}^{*}$, the ADC aspect thus increases the sensitivity of the layer spacing to changes in molecule tilt. While a negligible layer shrinkage is still a strong indication of ADC behaviour, we conclude that it is not a requirement.

X-ray scattering profiles from bulk samples of W504 do not contain any (001) x-ray 
scattering peak. This highly unusual feature can be understood by considering that the combination of one fluorinated tail and one ordinary alkoxy tail, taking the head-tail symmetry of the bulk liquid crystal phase into account, leads to a very weak electron density modulation along the smectic layer normal, and thus a vanishing form factor. The head-tail symmetry is however broken at the liquid crystal-air and liquid crystal-substrate interfaces, hence a thin film of W504 on an OTE-SAM substrate, examined in reflection, exhibits a nonzero form factor. Consequently, a (001) scattering peak is then observed in the scattering profile, allowing for the determination of the smectic layer spacing.

\section{ACKNOWLEDGMENTS}

J.L. gratefully acknowledges financial support from the Alexander von Humboldt Foundation and from the Swedish Research Council.

[1] F. Giesselmann, P. Zugenmaier, I. Dierking, S. T. Lagerwall, B. Stebler, M. Kaspar, V. Hamplova, and M. Glogarova, Phys. Rev. E. 60, 598 (1999).

[2] M. D. Radcliffe, M. L. Brostrom, K. A. Epstein, A. G. Rappaport, B. N. Thomas, R. F. Shao, and N. A. Clark, Liq. Cryst. 26, 789 (1999).

[3] Y. P. Panarin, V. Panov, O. Kalinovskaya, and J. K. Vij, J. Mater. Chem. 9, 2967 (1999).

[4] M. S. Spector, P. A. Heiney, J. Naciri, B. T. Weslowski, D. B. Holt, and R. Shashidhar, Phys. Rev. E 61, 1579 (2000).

[5] J. V. Selinger, P. J. Collings, and R. Shashidhar, Phys. Rev. E 64, 061705/1 (2001).

[6] N. A. Clark, T. Bellini, R. Shao, D. Coleman, S. Bardon, D. R. Link, J. E. Maclennan, X. H. Chen, M. D. Wand, D. M. Walba, et al., Appl. Phys. Lett. 80, 4097 (2002).

[7] F. Giesselmann, J. P. F. Lagerwall, G. Andersson, and M. D. Radcliffe, Phys. Rev. E. 66, 051704 / 1 (2002).

[8] J. P. F. Lagerwall, F. Giesselmann, and M. D. Radcliffe, Phys. Rev. E. 66, 031703 (2002).

[9] P. Collings, B. Ratna, and R. Shashidhar, Phys. Rev. E. 67, 021705 (2003).

[10] S. T. Wang, X. F. Han, Z. Q. Liu, A. Cady, M. D. Radcliffe, and C. C. Huang, Phys. Rev. E. 68, 060702(R) (2003). 
[11] J. P. F. Lagerwall, F. Giesselmann, A. Saipa, and R. Dabrowski, Liq. Cryst. 31, 1175 (2004).

[12] M. Rssle, R. Zentel, J. Lagerwall, and F. Giesselmann, Liq. Cryst. 31, 883 (2004).

[13] C. Huang, S. Wang, X. Han, A. Cady, R. Pindak, W. Caliebe, K. Ema, K. Takekoshi, and H. Yao, Phys. Rev. E. 69, 041702 (2004).

[14] M. Rssle, L. Braun, D. Schollmeyer, R. Zentel, J. P. F. Lagerwall, F. Giesselmann, and R. Stannarius, Liquid Crystals (accepted) (2005).

[15] R. B. Meyer, L. Liebert, L. Strzelecki, and P. Keller, J. Phys. Lett. 36, L69 (1975).

[16] A. De Vries, J. Chem. Phys. 71, 25 (1979).

[17] A. De Vries, Mol. Cryst. Liq. Cryst. Lett. 49, 179 (1979).

[18] A. De Vries, A. Ekachai, and N. Spielberg, Mol. Cryst. Liq. Cryst. Lett. 49, 143 (1979).

[19] A. de Vries, in Advances in Liquid Crystal Research and Applications, edited by L. Bata (Pergamon Press, Oxford, Budapest, 1980), pp. 71-80.

[20] M. Krueger and F. Giesselmann, Submitted to Phys. Rev. E. (2004).

[21] A. Saipa and F. Giesselmann, Liq. Cryst. 29, 347 (2002).

[22] K. Miyasato, S. Abe, H. Takezoe, and A. Fukuda, Jpn. J. Appl. Phys. Lett. 22, L661 (1983).

[23] M. Radcliffe, Personal communication (2002).

[24] J. Schacht, H. Baethge, F. Giesselmann, and P. Zugenmaier, J. Mater. Chem. 8, 603 (1998).

[25] P. Davidson, D. Petermann, and A. M. Levelut, J. Phys. (Paris) II 5, 113 (1995). 


\section{Figure captions}

1. The molecule under investigation, $(S)$-W504. The length is the largest core-core distance resulting from conformational energy minimization with MOPAC / AM1, plus one $\mathrm{F}$ and one $\mathrm{H}$ van der Waals radius.

2. Textures of W504 in four different cells. In the left and right columns the sample has been aligned for maximum extinction (within some uniformly aligned region in c, d, f, $\mathrm{i}$ and l) in the $\mathrm{SmA}^{*}$ and in the $\mathrm{SmC}^{*}$ phase, respectively. The centre column shows the $\mathrm{SmC}^{*}$ texture with the sample aligned as in the left column. The $\mathrm{SmA}^{*}$ pictures are taken at $T=130^{\circ}$ and the $\mathrm{SmC}^{*}$ pictures at $T=105^{\circ}$.

3. Optical director tilt angle $\theta(\mathrm{a})$, birefringence $\Delta n$ (b) and spontaneous polarization $P_{s}$ (c) as a function of temperature, the two former measured at $633 \mathrm{~nm}$ wavelength on a $1.5 \mu \mathrm{m}$ cell in the absence of field and with an electric field ensuring saturated switching applied.

4. Dielectric absorption spectra from W504 in a $23.5 \mu \mathrm{m}$ planar-aligned cell (a and b) at two amplitudes of the measuring field. $20 \mathrm{mV} / \mu \mathrm{m}$ is enough to unwind the helix in the $\mathrm{SmC}^{*}$ phase, as seen in the two examples of texture photos taken during the measurement. The helix unwinding leads to the large increase in $\mathrm{SmC}^{*}$ absorption in (b). As comparison, the corresponding dielectric spectrum of $(S)$-S1B8, an ordinary non-ADC FLC with $P_{s}$ similar to that of W504, is shown (c) with the same axis scalings as for W504. The $\mathrm{SmA}^{*} \mathrm{C}^{*}$ transition is at $119^{\circ}$ in $\mathrm{S} 1 \mathrm{~B} 8$, at $121.5^{\circ}$ in $\mathrm{W} 504$.

5. Small-angle X-ray scattering profiles from a non-aligned sample investigated with $\mathrm{Cu}-K_{\alpha}$ radiation (a) and from an OTE-SAM-aligned sample investigated at the synchrotron source (b), of W504 in $\operatorname{SmA}^{*}\left(140 / 139^{\circ}\right)$ and in $\mathrm{SmC}^{*}\left(110 / 111^{\circ}\right)$. From the synchrotron data the smectic layer spacing $d$ as a function of temperature $T$ was extracted (c).

6. WAXS scattering profile (rings) in $\mathrm{SmA}^{*}$ (a) and $\mathrm{SmC}^{*}(\mathrm{~b})$, the corresponding best fits of Eq. 2 (black curve) and the resulting average molecular inclination with respect to the smectic layer normal $\left\langle\theta_{m o l}\right\rangle$. For the $\mathrm{SmA}^{*}$ phase the orientational order parameter $S_{2}$ can also be estimated. 
Figures 


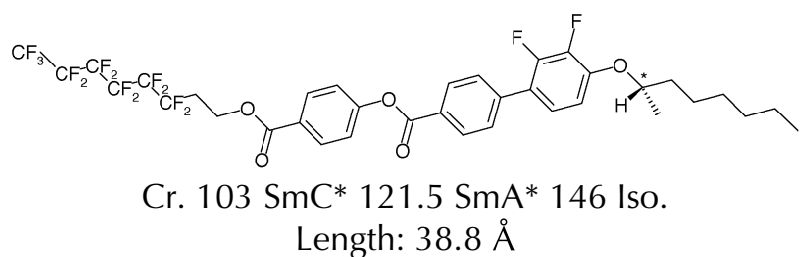

FIG. 1: 


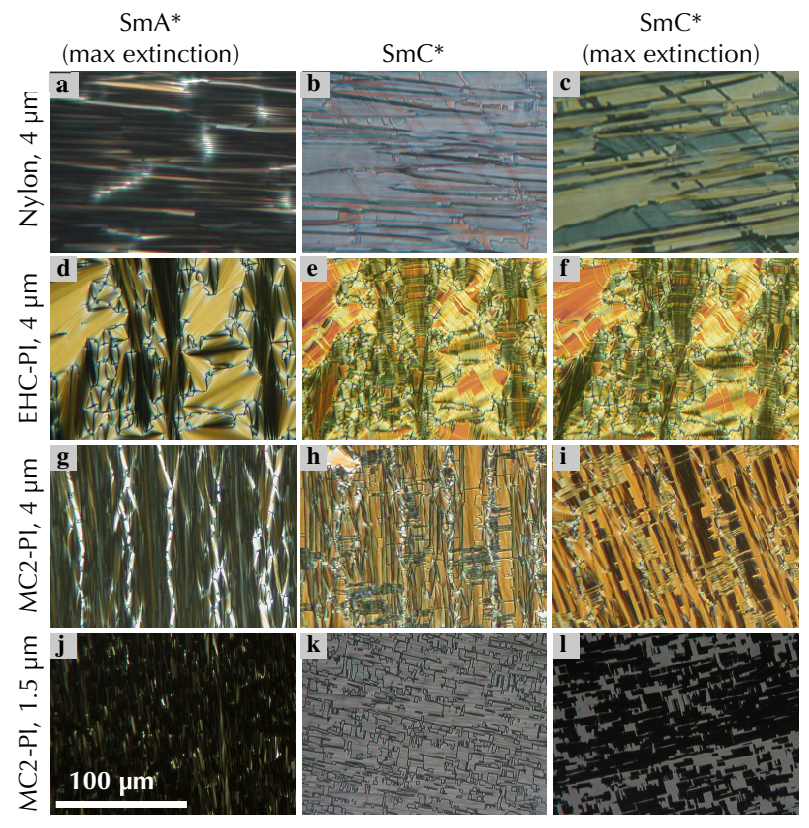

FIG. 2: 


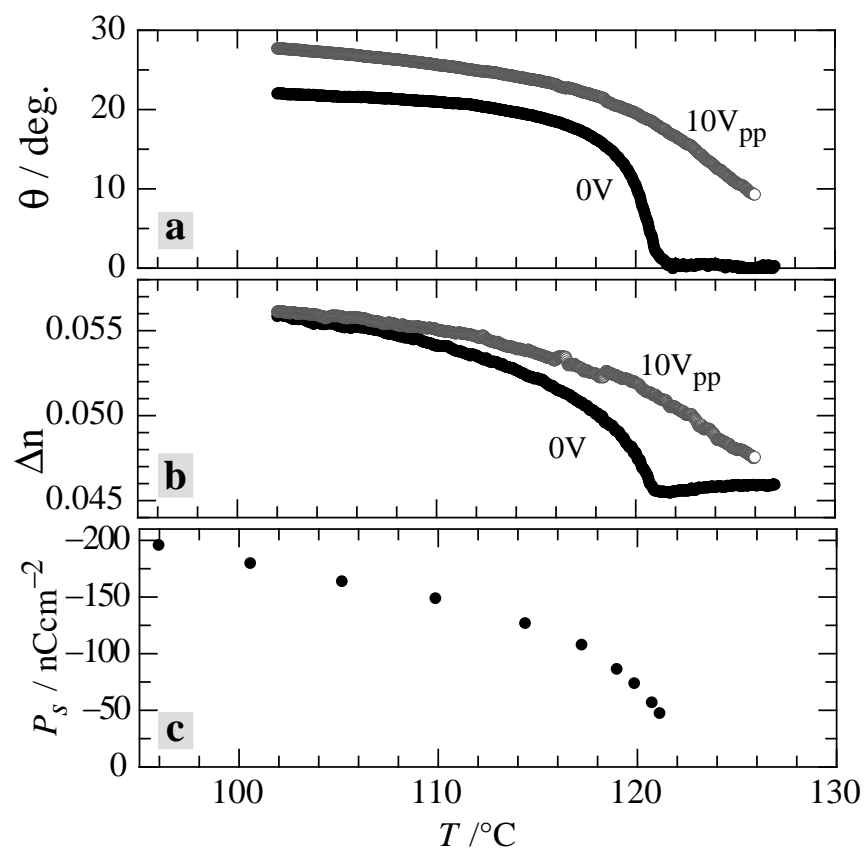

FIG. 3: 

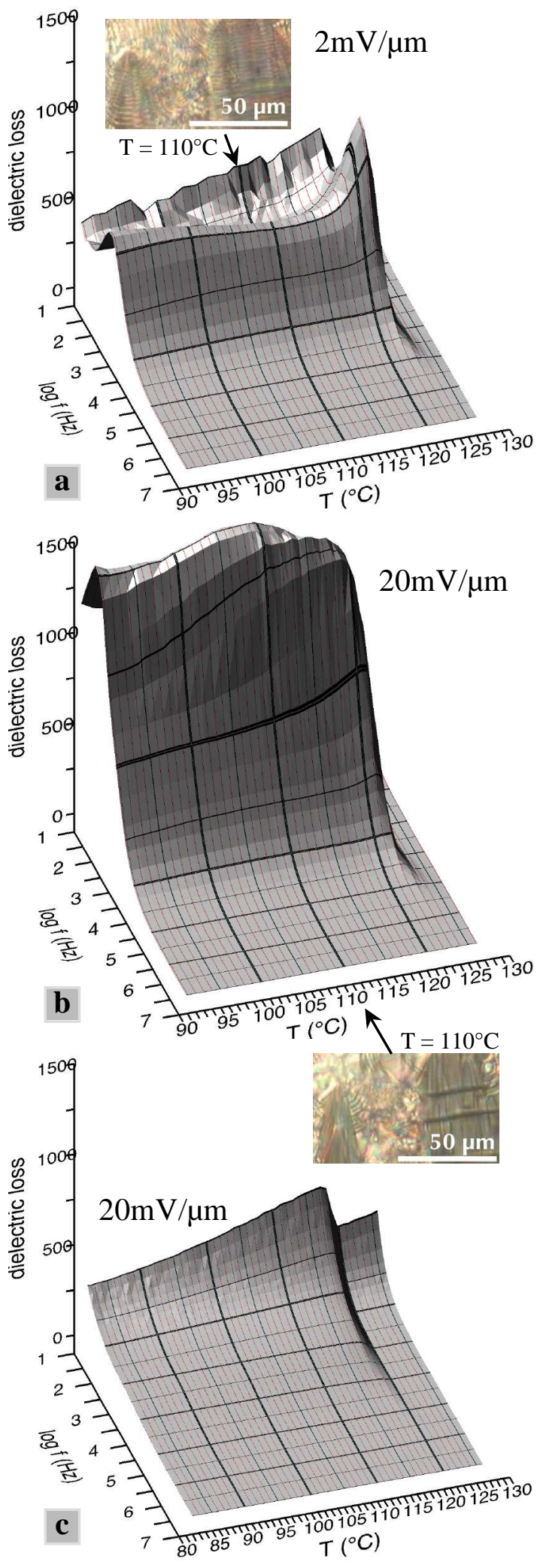

FIG. 4: 

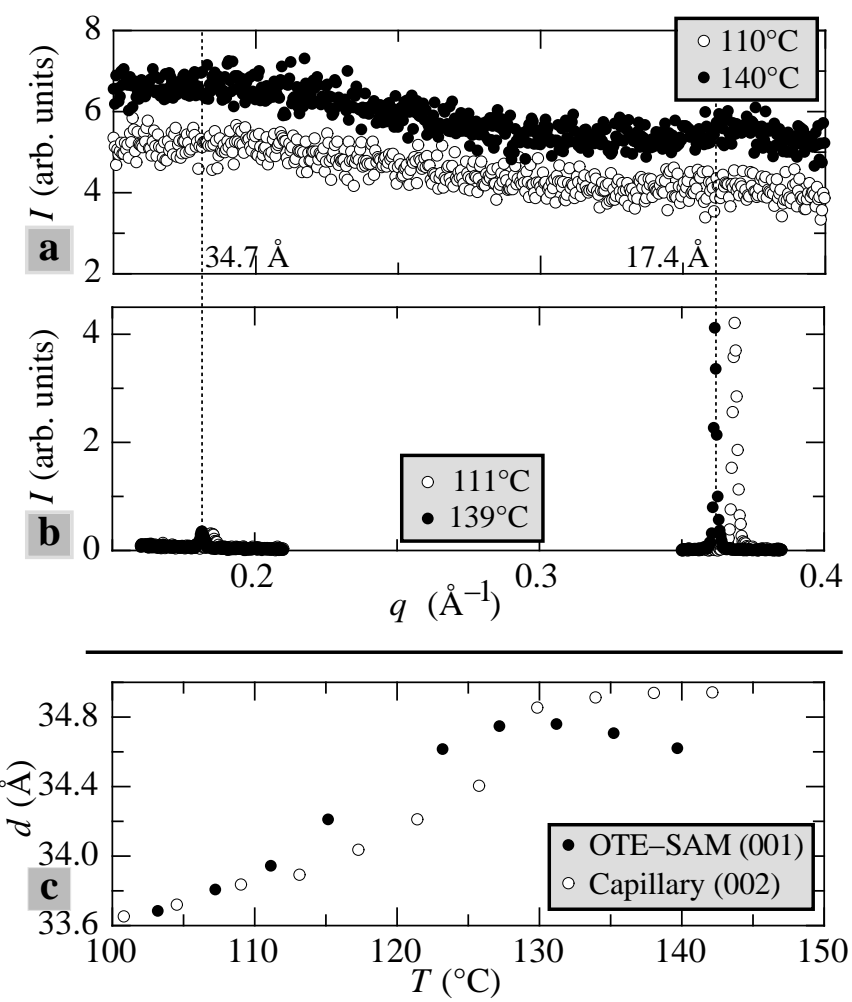

FIG. 5: 


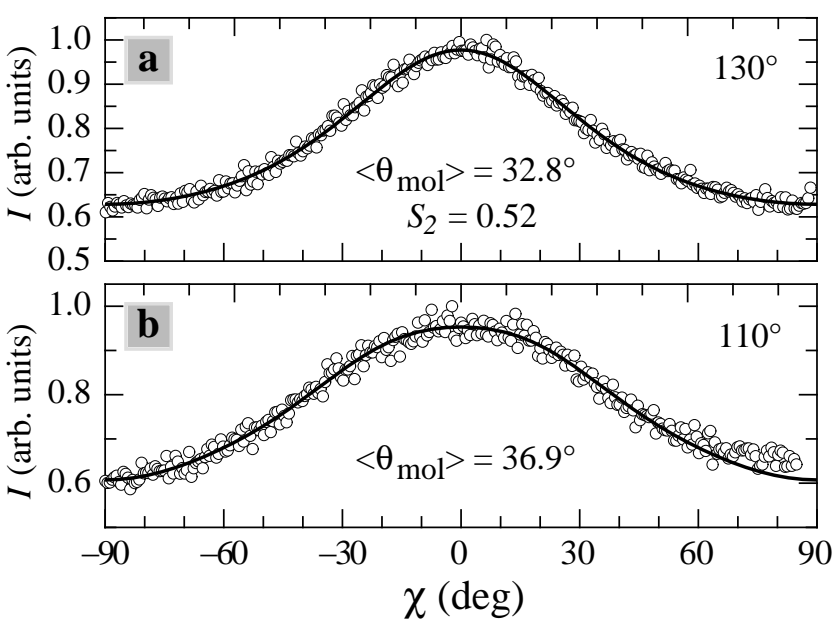

FIG. 6: 